\title{
10. CHEMICAL ANALYSES OF INTERLABORATORY STANDARDS
}

\author{
T.L. Wright, U.S. Geological Survey, Reston, Virginia
}

Four samples of basalt from Holes 332A and 332B were designated as standards to be analyzed by all investigators working on chemistry of Leg 37 basalts. In addition, wet chemical analyses of each standard were obtained from the U.S. Geological Survey's Denver rock analysis laboratory and from the U.S. National Museum. The analyses for major elements reported by all investigators are shown in Table 1.

For purposes of comparison each analysis was corrected for $\mathrm{CO}_{2}$ and normalized to dry weight. Standards 1,2 , and 3 were corrected by calculating $\mathrm{CO}_{2}$ as calcite and adjusting $\mathrm{CaO}$ accordingly. Standard 4 contains iron and magnesian carbonates and was corrected for a mixed carbonate consisting of $91 \% \mathrm{MgCO}_{3}$ and $9 \%$ $\mathrm{FeCO}_{3}$. After normalization ferric iron was converted to ferrous and all iron was expressed as "FeO." Where two iron values and/or $\mathrm{CO}_{2}$ were not reported, an average value for $\mathrm{Fe}_{2} \mathrm{O}_{3}$ and $\mathrm{CO}_{2}$ was assigned based on values given by the other laboratories. All adjusted and recalculated analyses are shown in Table 2 .

The interlaboratory agreement is good in general although small but consistent differences are apparent when comparing the results of any two laboratories for the four standards. Analyses of Standards 1-3 are plotted on $\mathrm{MgO}$ variation diagrams in Figure 1 . The total range of interlaboratory variation exceeds in some cases the variation found within single lithologic units analyzed on shipboard. This makes it difficult to make consistent chemical classifications when using analyses from different laboratories.

TABLE 1

Analyses of Interlaboratory Standards

\begin{tabular}{|c|c|c|c|c|c|c|c|c|c|}
\hline & USGS & USNM & BREST & DAL & USSR & URI & WOODS & R-UB & IMP \\
\hline \multicolumn{10}{|c|}{ Standard 1: Sample 332A-8-2, 2-15 cm } \\
\hline $\mathrm{SiO}_{2}$ & 50.93 & 50.69 & 50.08 & 50.77 & 50.14 & 50.86 & 51.51 & 51.08 & 50.90 \\
\hline $\mathrm{Al}_{2} \mathrm{O}_{3}$ & 14.60 & 15.23 & 14.26 & 14.79 & 14.54 & 14.50 & 14.60 & 14.42 & 14.40 \\
\hline $\mathrm{Fe}_{2} \mathrm{O}_{3}$ & 3.04 & 3.08 & 3.07 & 2.64 & 3.60 & 3.01 & 2.89 & 3.09 & 3.47 \\
\hline $\mathrm{FeO}$ & 7.04 & 7.02 & $6.92^{\mathrm{a}}$ & 7.41 & 7.26 & 7.19 & 7.11 & 7.00 & 7.00 \\
\hline $\mathrm{MgO}$ & 7.20 & 7.15 & 7.32 & 7.33 & 7.35 & 7.23 & 7.53 & 7.27 & 7.50 \\
\hline $\mathrm{CaO}$ & 12.07 & 12.22 & 12.25 & 12.24 & 12.10 & 12.24 & 11.99 & 11.98 & 12.10 \\
\hline $\mathrm{Na}_{2} \mathrm{O}$ & 2.24 & 2.13 & 2.23 & 2.21 & 2.24 & 2.23 & 2.17 & 2.14 & 2.23 \\
\hline $\mathrm{K}_{2} \mathrm{O}$ & 0.32 & 0.28 & 0.33 & 0.32 & 0.35 & 0.34 & 0.33 & 0.32 & 0.31 \\
\hline $\mathrm{H}_{2} \mathrm{O}$ & 0.82 & 0.82 & 0.82 & 1.00 & 0.89 & 0.91 & 0.93 & 1.10 & 1.10 \\
\hline $\mathrm{TiO}_{2}$ & 1.17 & 1.18 & 1.10 & 1.11 & 1.20 & 1.11 & 1.08 & 1.08 & 1.12 \\
\hline $\mathrm{P}_{2} \mathrm{O}_{5}$ & 0.12 & 0.08 & 0.12 & 0.11 & $0.0^{\mathrm{c}}$ & 0.13 & $0.0^{\mathrm{e}}$ & 0.13 & 0.12 \\
\hline $\mathrm{MnO}$ & 0.17 & 0.18 & 0.16 & 0.18 & 0.20 & 0.18 & 0.15 & 0.17 & 0.18 \\
\hline $\mathrm{CO}_{2}$ & 0.11 & 0.09 & $0.11^{\mathrm{b}}$ & 0.14 & 0.09 & $0.11^{\mathrm{b}}$ & 0.06 & $<0.10$ & $<0.10$ \\
\hline \multirow[t]{2}{*}{ Total } & 99.93 & 100.20 & 98.77 & 100.25 & 99.96 & 100.04 & 100.35 & 99.88 & 100.53 \\
\hline & USGS & USNM & BREST & USSR & GUNN & WOODS & R-UB & IMP & \\
\hline \multicolumn{10}{|c|}{ Standard 2: Sample 332A-40-2, 26-33 cm } \\
\hline $\mathrm{SiO}_{2}$ & 47.72 & 47.42 & 47.50 & 47.40 & 48.83 & 48.08 & 47.94 & 47.42 & \\
\hline $\mathrm{Al}_{2} \mathrm{O}_{3}$ & 17.45 & 17.82 & 17.17 & 17.24 & 16.82 & 17.77 & 16.88 & 17.52 & \\
\hline $\mathrm{Fe}_{2} \mathrm{O}_{3}$ & 1.83 & 2.12 & $2.23^{\mathrm{a}}$ & 2.75 & $2.23^{\mathrm{a}}$ & 2.08 & 1.75 & 1.57 & \\
\hline $\mathrm{FeO}$ & 4.57 & 4.41 & 4.17 & 4.10 & 4.43 & 4.34 & 4.60 & 4.90 & \\
\hline $\mathrm{MgO}$ & 9.25 & 9.18 & 9.12 & 9.25 & 9.39 & 9.02 & 9.31 & 9.42 & \\
\hline $\mathrm{CaO}$ & 15.10 & 15.34 & 15.46 & 15.00 & 15.49 & 14.99 & 14.82 & 15.38 & \\
\hline $\mathrm{Na}_{2} \mathrm{O}$ & 1.51 & 1.42 & 1.46 & 1.57 & 1.48 & 1.58 & 1.64 & 1.50 & \\
\hline $\mathrm{K}_{2} \mathrm{O}$ & 0.12 & 0.08 & 0.11 & 0.11 & 0.11 & 0.09 & 0.06 & 0.10 & \\
\hline $\mathrm{H}_{2} \mathrm{O}$ & 0.63 & 0.90 & 1.86 & 0.87 & 0.0 & .0 .87 & 0.40 & 0.40 & \\
\hline $\mathrm{TiO}_{2}$ & 0.55 & 0.58 & 0.55 & 0.63 & 0.55 & 0.53 & 0.53 & 0.54 & \\
\hline $\mathrm{P}_{2} \mathrm{O}_{5}$ & 0.06 & 0.05 & 0.07 & $0.0^{c}$ & 0.07 & $0.0^{c}$ & 0.10 & 0.05 & \\
\hline $\mathrm{MnO}$ & 0.12 & 0.14 & 0.12 & 0.14 & 0.12 & 0.12 & 0.10 & 0.12 & \\
\hline $\mathrm{CO}_{2}$ & 0.96 & 0.98 & $0.97^{\mathrm{b}}$ & 0.96 & $0.97^{\mathrm{b}}$ & 0.86 & 0.68 & 0.68 & \\
\hline Total & 99.87 & 100.44 & 100.79 & 100.02 & 100.49 & 100.83 & 98.81 & 99.60 & \\
\hline
\end{tabular}


TABLE 1 - Continued

\begin{tabular}{|c|c|c|c|c|c|c|c|c|c|c|}
\hline & USGS & USNM & BREST & DAL & USSR & GUNN & URI & WOODS & R-UB & IMP \\
\hline \multicolumn{11}{|c|}{ Standard 3: Sample 332B-3-4, 26-36 cm } \\
\hline $\mathrm{SiO}_{2}$ & 48.46 & 48.26 & 48.50 & 47.47 & 48.14 & 49.15 & 48.40 & 48.60 & 48.54 & 48.38 \\
\hline $\mathrm{Al}_{2} \mathrm{O}_{3}$ & 20.18 & 20.53 & 20.22 & 20.44 & 19.74 & 19.46 & 19.81 & 19.59 & 19.60 & 20.02 \\
\hline $\mathrm{Fe}_{2} \mathrm{O}_{3}$ & 1.39 & 1.51 & $1.47^{\mathrm{a}}$ & 1.22 & 1.90 & $1.47^{\mathrm{a}}$ & 1.34 & 1.29 & 1.27 & 1.50 \\
\hline $\mathrm{FeO}$ & 4.55 & 4.46 & 4.57 & 4.84 & 4.56 & 4.62 & 4.75 & 4.82 & 4.65 & 4.65 \\
\hline $\mathrm{MgO}$ & 7.66 & 7.59 & 7.75 & 7.73 & 7.60 & 7.73 & 7.55 & 8.16 & 7.74 & 7.92 \\
\hline $\mathrm{CaO}$ & 14.83 & 14.95 & 14.90 & 15.14 & 14.88 & 15.00 & 14.95 & 14.61 & 14.51 & 14.80 \\
\hline $\mathrm{Na}_{2} \mathrm{O}$ & 1.54 & 1.47 & 1.37 & 1.49 & 1.60 & 1.46 & 1.55 & 1.60 & 1.58 & 1.55 \\
\hline $\mathrm{K}_{2} \mathrm{O}$ & 0.05 & 0.05 & 0.06 & 0.05 & 0.05 & 0.05 & 0.08 & 0.06 & 0.08 & 0.05 \\
\hline $\mathrm{H}_{2} \mathrm{O}$ & 0.66 & 0.67 & 0.63 & 0.60 & 0.58 & 0.0 & 0.59 & 0.68 & 0.70 & 0.70 \\
\hline $\mathrm{TiO}_{2}$ & 0.41 & 0.43 & 0.42 & 0.41 & 0.50 & 0.41 & 0.42 & 0.40 & 0.40 & 0.42 \\
\hline $\mathrm{P}_{2} \mathrm{O}_{5}$ & 0.04 & 0.03 & 0.03 & 0.03 & $0.0^{\mathrm{c}}$ & 0.04 & 0.03 & $0.0^{\mathrm{c}}$ & 0.05 & 0.04 \\
\hline $\mathrm{MnO}$ & 0.10 & 0.12 & 0.10 & 0.11 & 0.12 & 0.11 & 0.10 & 0.11 & 0.12 & 0.11 \\
\hline $\mathrm{CO}_{2}$ & 0.04 & 0.02 & $0.02^{\mathrm{b}}$ & 0.01 & $0.02^{b}$ & $0.02^{b}$ & $0.02^{\mathrm{b}}$ & 0.07 & $<0.10$ & $<0.10$ \\
\hline \multirow[t]{2}{*}{ Total } & 99.91 & 100.90 & 100.04 & 99.54 & 99.69 & 99.52 & 99.59 & 99.99 & 99.34 & 100.24 \\
\hline & USGS & USNM & BREST & USSR & GUNN & URI & WOODS & R-UB & IMP & \\
\hline \multicolumn{11}{|c|}{ Standard 4: Sample 332B-35-2, 51-58 cm } \\
\hline $\mathrm{SiO}_{2}$ & 44.44 & 44.14 & 44.08 & 44.20 & 46.10 & 44.22 & 44.89 & 44.14 & 44.36 & \\
\hline $\mathrm{Al}_{2} \mathrm{O}_{3}$ & 11.96 & 12.37 & 11.35 & 11.71 & 11.91 & 11.67 & 11.82 & 11.51 & 12.32 & \\
\hline $\mathrm{Fe}_{2} \mathrm{O}_{3}$ & 2.76 & 3.00 & $2.99^{\mathrm{a}}$ & 3.45 & $2.99^{\mathrm{a}}$ & 2.74 & 2.61 & 3.08 & 2.92 & \\
\hline $\mathrm{FeO}$ & 6.46 & 6.32 & 6.45 & 6.51 & 6.95 & 6.60 & 6.49 & 6.30 & 6.30 & \\
\hline $\mathrm{MgO}$ & 18.99 & 18.89 & 19.16 & 19.25 & 19.48 & 19.16 & 19.34 & 19.14 & 18.99 & \\
\hline $\mathrm{CaO}$ & 8.68 & 8.84 & 8.43 & 8.35 & 9.25 & 8.66 & 8.82 & 8.76 & 8.72 & \\
\hline $\mathrm{Na}_{2} \mathrm{O}$ & 1.55 & 1.41 & 1.53 & 1.52 & 1.69 & 1.60 & 1.53 & 1.45 & 1.53 & \\
\hline $\mathrm{K}_{2} \mathrm{O}$ & 0.18 & 0.15 & 0.19 & 0.16 & 0.18 & 0.17 & 0.18 & 0.20 & 0.17 & \\
\hline $\mathrm{H}_{2} \mathrm{O}$ & 1.82 & 2.22 & 4.18 & 2.24 & 0.0 & 1.56 & 2.64 & 2.10 & 2.10 & \\
\hline $\mathrm{TiO}_{2}$ & 0.44 & 0.46 & 0.46 & 0.48 & 0.47 & 0.47 & 0.43 & 0.45 & 0.46 & \\
\hline $\mathrm{P}_{2} \mathrm{O}_{5}$ & 0.04 & 0.04 & 0.04 & $0.0^{\mathrm{c}}$ & 0.05 & 0.04 & $0.0^{\mathrm{c}}$ & 0.04 & 0.04 & \\
\hline $\mathrm{MnO}$ & 0.15 & 0.16 & 0.15 & 0.21 & 0.16 & 0.15 & 0.15 & 0.16 & 0.16 & \\
\hline $\mathrm{CO}_{2}$ & 2.37 & 2.32 & $2.30^{\mathrm{b}}$ & 2.20 & $2.30^{\mathrm{b}}$ & $2.30^{\mathrm{b}}$ & 2.21 & 2.01 & 2.01 & \\
\hline Total & 99.84 & 100.32 & 101.31 & 100.28 & 101.53 & 99.34 & 101.11 & 99.34 & 100.08 & \\
\hline
\end{tabular}

Note: USGS $=$ U. S. Geological Survey Denver Rock Analysis Laboratory. Method $=$ wet chemical. Analyst $=$ E. Engleman. USNM $=$ U. S. National Museum. Method $=$ wet chemical. Analyst $=$ J. Norbert. BREST $=$ Centre Nationale Pour Exploitation des Oceans. Method $=$ X-ray fluorescence $(\mathrm{XRF})$. Analyst $=$ Pierre Cambon. $\mathrm{DAL}=$ Rock analysis laboratory, Dalhousie University. Method: $\mathrm{SiO}_{2}-$ Fast neutron activation; $\mathrm{CO}_{2}=$ automatic lead titrator; $\mathrm{TiO}_{2}$ and $\mathrm{P}_{2} \mathrm{O}_{5}-$ calorimetric; $\mathrm{H}_{2} \mathrm{O}^{+}$and $\mathrm{H}_{2} \mathrm{O}^{-}$- gravimetrically; $\mathrm{FeO}$ - modified cold acid decomposition method of Wilson. All remaining oxides done by atomic absorbtion spectroscopy (AAS) after dissolution with $\mathrm{HF} / \mathrm{HClO}_{4}$. Analyst $=\mathrm{P}$. Jagam, S. Parikh, V. Murphy, and M. Fratta. USSR = Institution of Geochemistry, USSR Academy of Science. Method $=$ wet chemical (A. I. Ponomarev. The methods of the chemical analyses of the silicate and carbonate rocks. Academy of Sciences, Moscow, 1961). Analyst $=$ A. V. Lebedkova. URI = University of Rhode Island. Method $=\mathrm{SiO}_{2}, \mathrm{H}_{2} \mathrm{O}-$ gravimetric; $\mathrm{TiO}_{2}, \mathrm{P}_{2} \mathrm{O}_{5}$; Total iron as $\mathrm{Fe}_{2} \mathrm{O}_{3}$ - colorimetric; $\mathrm{FeO}$ - ration; all other oxides by atomic absorbtion spectroscopy (AAS). Analyst $=\mathrm{R}$. Evans. WOODS $=$ Woods Hole Oceanographic Institution. Method $=$ fusion and microprobe spectroscopy; $\mathrm{FeO}$ by titrimetry; $\mathrm{H}_{2} \mathrm{O}$ and $\mathrm{CO}_{2}$ by gas chromatography. Analyst $=\mathrm{G}$. Thompson. GUNN $=$ Universite de Montreal. Method $=\mathrm{X}$-ray fluorescence $(\mathrm{XRF})$. Analyst $=\mathrm{B}$. Gunn. $\mathrm{R}-\mathrm{UB}=$ Ruhr-Universität Bochum. Method $=\mathrm{X}$-ray fluorescence $(\mathrm{XRF}) ; \mathrm{Na}_{2} \mathrm{O}, \mathrm{K}_{2} \mathrm{O}, \mathrm{MnO}-$ atomic absorbtion spectroscopy (AAS); $\mathrm{FeO}$ - titration; $\mathrm{H}_{2} \mathrm{O}$ - instrumental. Analysts = $\mathrm{P}$. Robinson and $\mathrm{H}$. Henning. $\mathrm{CO}_{2}$ determinations by $\mathrm{H}$. Puchelt, Universitat Karlsruhe. $\mathrm{IMP}=$ Imperial College, London. Method $=\mathrm{X}$-ray fluorescence $(\mathrm{XRF}) ; \mathrm{Na}_{2} \mathrm{O}$ - flame photometry; $\mathrm{FeO}-$ titration; $\mathrm{H}_{2} \mathrm{O}-$ instrumental. Analysts $=$ M. F. J. Flower and $\mathrm{H}$. Henning. $\mathrm{CO}_{2}$ determinations by H. Puchelt, Universität Karlsruhe.

${ }^{\mathrm{a}}$ Total iron reported as $\mathrm{Fe}_{2} \mathrm{O}_{3}$ is average value assigned based on values given by the other laboratories.

${ }^{\mathrm{b}} \mathrm{CO}_{2}$ not reported. $\mathrm{CO}_{2}$ shown is average value based on values given by the other laboratories.

${ }^{\mathrm{c}} \mathrm{P}_{2} \mathrm{O}_{5}$ not reported. 
TABLE 2

Adjusted Analyses of Interlaboratory Standards

\begin{tabular}{lrrrrrrrrr}
\hline \multicolumn{2}{c}{ USGS } & USNM & BREST & DAL & USSR & URI & WOODS & R-UB & IMP \\
\hline \multicolumn{2}{l}{$\mathrm{Standard}^{1}$} & & & & & & & & \\
$\mathrm{SiO}_{2}$ & 51.57 & 51.16 & 51.26 & 51.32 & 50.72 & 51.44 & 51.88 & 51.83 & 51.31 \\
$\mathrm{Al}_{2} \mathrm{O}_{3}$ & 14.78 & 15.37 & 14.60 & 14.95 & 14.71 & 14.66 & 14.71 & 14.63 & 14.52 \\
"FeO" & 9.90 & 9.88 & 9.91 & 9.89 & 10.62 & 10.01 & 9.78 & 9.92 & 10.20 \\
$\mathrm{MgO}$ & 7.29 & 7.22 & 7.49 & 7.41 & 7.43 & 7.31 & 7.58 & 7.38 & 7.56 \\
$\mathrm{CaO}$ & 12.08 & 12.22 & 12.39 & 12.19 & 12.12 & 12.24 & 12.00 & 12.03 & 12.07 \\
$\mathrm{Na}_{2} \mathrm{O}$ & 2.27 & 2.15 & 2.28 & 2.23 & 2.27 & 2.26 & 2.19 & 2.17 & 2.25 \\
$\mathrm{~K}_{2} \mathrm{O}$ & 0.32 & 0.28 & 0.34 & 0.32 & 0.35 & 0.34 & 0.33 & 0.32 & 0.31 \\
$\mathrm{TiO}_{2}$ & 1.18 & 1.14 & 1.13 & 1.12 & 1.21 & 1.12 & 1.09 & 1.10 & 1.13 \\
$\mathrm{P}_{2} \mathrm{O}_{5}$ & 0.12 & 0.08 & 0.12 & 0.11 & 0.0 & 0.13 & 0.0 & 0.13 & 0.12 \\
$\mathrm{MnO}$ & 0.17 & 0.18 & 0.16 & 0.18 & 0.20 & 0.18 & 0.15 & 0.17 & 0.18 \\
$\mathrm{Total}^{\mathrm{a}}$ & 99.69 & 99.69 & 99.69 & 99.73 & 99.64 & 99.70 & 99.71 & 99.69 & 99.65
\end{tabular}

\begin{tabular}{|c|c|c|c|c|c|c|c|c|c|c|}
\hline & & USGS & USNM & BREST & USSR & GUNN & WOODS & R-UB & IMP & \\
\hline & \multicolumn{10}{|c|}{ Standard 2} \\
\hline & $\mathrm{SiO}_{2}$ & 49.17 & 48.73 & 49.11 & 48.88 & 49.68 & 49.31 & 49.49 & 48.56 & \\
\hline & $\mathrm{Al}_{2} \mathrm{O}_{3}$ & 17.98 & 18.31 & 17.75 & 17.78 & 17.11 & 18.22 & 17.43 & 17.94 & \\
\hline & "FeO" & 6.41 & 6.49 & 6.39 & 6.78 & 6.55 & 6.37 & 6.38 & 6.47 & \\
\hline & $\mathrm{MgO}$ & 9.53 & 9.43 & 9.43 & 9.54 & 9.55 & 9.25 & 9.61 & 9.65 & \\
\hline & $\mathrm{CaO}$ & 14.30 & 14.48 & 14.71 & 14.21 & 14.50 & 14.25 & 14.41 & 14.86 & \\
\hline & $\mathrm{Na}_{2} \mathrm{O}$ & 1.56 & 1.46 & 1.51 & 1.52 & 1.61 & 1.62 & 1.69 & 1.54 & \\
\hline & $\mathrm{K}_{2} \mathrm{O}$ & 0.12 & 0.08 & 0.11 & 0.11 & 0.11 & 0.09 & 0.06 & 0.10 & \\
\hline & $\mathrm{TiO}_{2}$ & 0.57 & 0.60 & 0.57 & 0.65 & 0.56 & 0.54 & 0.55 & 0.55 & \\
\hline & $\mathrm{P}_{2} \mathrm{O}_{5}$ & 0.06 & 0.05 & 0.07 & 0.0 & 0.07 & 0.0 & 0.10 & 0.05 & \\
\hline & $\mathrm{MnO}$ & 0.12 & 0.14 & 0.12 & 0.14 & 0.12 & 0.12 & 0.10 & 0.12 & \\
\hline & Total $^{\mathrm{a}}$ & 99.81 & 99.78 & 99.77 & 99.72 & 99.77 & 99.79 & 99.81 & 99.84 & \\
\hline & USGS & USNM & BREST & DAL & USSR & GUNN & URI & WOODS & R-UB & IMP \\
\hline \multicolumn{11}{|c|}{ Standard 3} \\
\hline $\mathrm{SiO}_{2}$ & 48.87 & 48.56 & 48.81 & 47.99 & 48.59 & 49.41 & 48.91 & 49.02 & 49.32 & 48.72 \\
\hline $\mathrm{Al}_{2} \mathrm{O}_{3}$ & 20.35 & 20.66 & 20.35 & 20.66 & 19.93 & 19.56 & 20.02 & 19.76 & 19.92 & 20.16 \\
\hline "FeO" & 5.85 & 5.86 & 5.93 & 6.00 & 6.33 & 5.97 & 6.02 & 6.03 & 5.89 & 6.04 \\
\hline $\mathrm{MgO}$ & 7.72 & 7.64 & 7.80 & 7.81 & 7.67 & 7.77 & 7.63 & 8.23 & 7.87 & 7.98 \\
\hline $\mathrm{CaO}$ & 14.90 & 15.02 & 14.97 & 15.29 & 14.99 & 15.05 & 15.08 & 14.65 & 14.61 & 14.77 \\
\hline $\mathrm{Na}_{2} \mathrm{O}$ & 1.55 & 1.48 & 1.38 & 1.51 & 1.62 & 1.47 & 1.57 & 1.61 & 1.61 & 1.56 \\
\hline $\mathrm{K}_{2} \mathrm{O}$ & 0.05 & 0.05 & 0.06 & 0.05 & 0.05 & 0.05 & 0.08 & 0.06 & 0.08 & 0.05 \\
\hline $\mathrm{TiO}_{2}$ & 0.41 & 0.43 & 0.42 & 0.41 & 0.50 & 0.41 & 0.42 & 0.40 & 0.40 & 0.42 \\
\hline $\mathrm{P}_{2} \mathrm{O}_{5}$ & 0.04 & 0.03 & 0.03 & 0.03 & 0.0 & 0.04 & 0.03 & 0.0 & 0.05 & 0.04 \\
\hline $\mathrm{MnO}$ & 0.10 & 0.12 & 0.10 & 0.11 & 0.12 & 0.11 & 0.10 & 0.11 & 0.12 & 0.11 \\
\hline Total $^{\mathrm{a}}$ & 99.86 & 99.85 & 99.85 & 99.88 & 99.81 & 99.85 & 99.86 & 99.87 & 99.87 & 99.85 \\
\hline
\end{tabular}

\begin{tabular}{lrrrrrrrrr}
\hline & USGS & USNM & BREST & USSR & GUNN & URI & WOODS & R-UB & IMP \\
\hline $\mathrm{Standard}^{4}$ & & & & & & & & & \\
$\mathrm{SiO}_{2}$ & 47.62 & 47.21 & 47.62 & 47.19 & 47.54 & 47.44 & 47.71 & 47.33 & 47.20 \\
$\mathrm{Al}_{2} \mathrm{O}_{3}$ & 12.82 & 13.23 & 12.26 & 12.50 & 12.28 & 12.52 & 12.56 & 12.34 & 13.11 \\
"FeO" & 9.19 & 9.26 & 9.49 & 9.90 & 9.57 & 9.34 & 9.03 & 9.39 & 9.17 \\
$\mathrm{MgO}$ & 18.24 & 18.15 & 18.64 & 18.60 & 18.12 & 18.51 & 18.61 & 18.74 & 18.43 \\
$\mathrm{CaO}$ & 9.30 & 9.46 & 9.11 & 8.91 & 9.54 & 9.29 & 9.37 & 9.39 & 9.28 \\
$\mathrm{Na}_{2} \mathrm{O}$ & 1.66 & 1.51 & 1.65 & 1.62 & 1.74 & 1.72 & 1.63 & 1.55 & 1.63 \\
$\mathrm{~K}_{2} \mathrm{O}$ & 0.19 & 0.16 & 0.21 & 0.17 & 0.19 & 0.18 & 0.19 & 0.21 & 0.18 \\
$\mathrm{TiO}_{2}$ & 0.47 & 0.49 & 0.50 & 0.51 & 0.48 & 0.50 & 0.46 & 0.48 & 0.49 \\
$\mathrm{P}_{2} \mathrm{O}_{5}$ & 0.04 & 0.04 & 0.04 & 0.0 & 0.05 & 0.04 & 0.0 & 0.04 & 0.04 \\
$\mathrm{MnO}^{2}$ & 0.16 & 0.17 & 0.16 & 0.22 & 0.17 & 0.16 & 0.16 & 0.17 & 0.17 \\
$\mathrm{Total}^{\mathrm{a}}$ & 99.70 & 99.68 & 99.68 & 99.63 & 99.69 & 99.71 & 99.72 & 99.67 & 99.69 \\
\hline
\end{tabular}

${ }^{\mathrm{a}}$ The deviation of the total from $100 \%$ reflects the conversion of $\mathrm{Fe}_{2} \mathrm{O}_{3}$ to $\mathrm{FeO}$. 

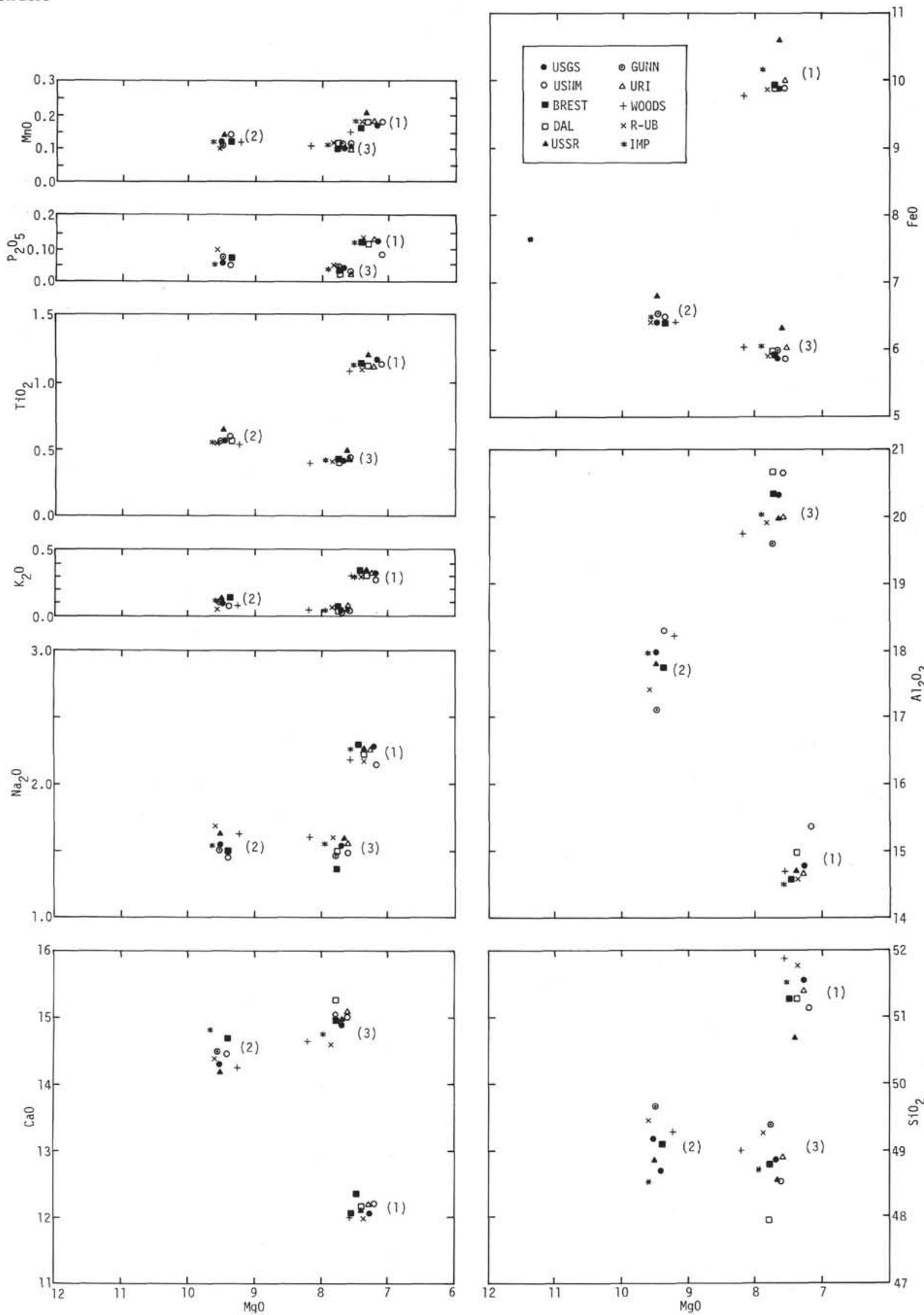

Figure 1. MgO variation diagrams for standard Samples 1,2, and 3. Data are from adjusted analyses of Table 2. Laboratories coded as follows: $\bullet$ USGS; ○ USNM; $\mathbf{B}$ BRST; $\square$ DAL; $\triangle$ USSR; $\odot$ GUNN; $\triangle U R I ;+W O O D S$; x $R-U B ; * I M P$. 